\title{
RAINBOW MATCHINGS IN PROPERLY COLORED MULTIGRAPHS*
}

\author{
PETER KEEVASH $^{\dagger}$ AND LIANA YEPREMYAN ${ }^{\dagger}$
}

\begin{abstract}
Aharoni and Berger conjectured that in any bipartite multigraph that is properly edge-colored by $n$ colors with at least $n+1$ edges of each color there must be a matching that uses each color exactly once. In this paper we consider the same question without the bipartiteness assumption. We show that in any multigraph with edge multiplicities $o(n)$ that is properly edgecolored by $n$ colors with at least $n+o(n)$ edges of each color there must be a matching of size $n-O(1)$ that uses each color at most once.
\end{abstract}

Key words. graph theory, matchings, latin squares

AMS subject classification. $05 \mathrm{C} 70$

DOI. $10.1137 / 17 \mathrm{M} 1151742$

1. Introduction. A Latin square of order $n$ is an $n \times n$ array of $n$ symbols in which each symbol occurs exactly once in each row and in each column. A (partial) transversal of a Latin square is a set of entries that represent each row, column, and symbol (at most) exactly once. The following fundamental open problem on transversals in Latin squares is known as the Ryser-Brualdi-Stein conjecture. ${ }^{1}$ (The best known bound is $n-O\left(\log ^{2} n\right)$ by Hatami and Shor [5].)

Conjecture 1.1 (Ryser-Brualdi-Stein). Every Latin square of order $n$ has a partial transversal of size $n-1$.

It is not hard to see that Conjecture 1.1 is equivalent to saying that any proper $n$-edge-coloring of the complete bipartite graph $K_{n, n}$ has a rainbow matching (i.e., a matching with no repeated color) of size $n-1$. Aharoni and Berger [1] conjectured the following generalization.

Conjecture 1.2 (Aharoni and Berger). Let $G$ be a bipartite multigraph that is properly edge-colored with $n$ colors and has at least $n+1$ edges of each color. Then $G$ has a rainbow matching using every color.

Pokrovskiy [6] showed that this conjecture is asymptotically true, in that the conclusion holds if there are at least $n+o(n)$ edges of each color. In this paper we consider the same question without the bipartiteness assumption. We obtain a result somewhat analogous to Pokrovskiy's, although we require an additional (mild) assumption on edge multiplicities (which is perhaps just an artifact of our proof), and we have to allow a constant number of unused colors. A bipartite version of our result (without the additional assumption on edge multiplicities) was obtained by Barát,

\footnotetext{
* Received by the editors October 12, 2017; accepted for publication (in revised form) May 23, 2018; published electronically July 10, 2018.

http://www.siam.org/journals/sidma/32-3/M115174.html

Funding: Research was supported in part by ERC Consolidator grant 647678 .

$\dagger$ Mathematical Institute, University of Oxford, Oxford, UK (keevash@maths.ox.ac.uk, yepremyan@maths.ox.ac.uk).

${ }^{1}$ Ryser [8] conjectured that the number of transversals has the same parity as $n$, so any Latin square of odd order has a transversal (see [10]). For any even $n$ the addition table of a cyclic group is a Latin square with no transversal. Brualdi made Conjecture 1.1 (see, e.g., [3]); Stein [9] independently made a stronger conjecture, recently disproved by Pokrovskiy and Sudakov [7].
} 
Gyárfás, and Sárközy [2]. ${ }^{2}$ We give explicit constants in the statement for the sake of concreteness, but we did not attempt to optimize these.

Theorem 1.1. Let $0<\varepsilon<10^{-3}, k=2^{20 / \varepsilon}$, and $n>k^{2}$. Suppose $G$ is a multigraph with edge multiplicities at most $n / k$ that is properly edge-colored with $n$ colors so that every color appears at least $(1+\varepsilon) n$ times. Then $G$ has a rainbow matching of size $n-k$.

Our proof of Theorem 1.1 is algorithmic. Given any rainbow matching $M$ of size less than $n-k$, we construct a hierarchy of edges $e$ in $M$ for which we can find another rainbow matching $M^{\prime}$ of the same size which does not contain $e$, or indeed any edge with the same color as $e$. We find $M^{\prime}$ by a sequence of switches using edges at lower levels in hierarchy. The switching method is robust, in that there are many choices at each step, and so it is possible to avoid any constant size set of vertices or edges that were altered by previous switches. To analyze the algorithm, we suppose for contradiction that it does not find a switch to increase the matching, and then prove several structural properties of $G$ that culminate in a counting argument to give the required contradiction.

We describe and analyze our algorithm in section 3, after briefly summarizing our notation in the next section. Then we give the proof of Theorem 1.1 in section 4 . In the conclusion we compare our work to a recent result of Gao et al. [4].

2. Notation. For convenient reference we summarize our notation in this section.

Let $G$ be a properly edge-colored multigraph. We write $c(e)$ for the color of an edge $e$. We also say that $e$ is a $c$-edge, where $c(e)=c$. Similarly, for any set $C$ of colors, we say that $e$ is a $C$-edge if $c(e) \in C$.

Now suppose $M$ is a rainbow matching in $G$. We let $C(M)$ denote the set of colors used by $M$. For $c \in C(M)$ we let $m_{c}$ be the edge of $M$ of color $c$. If $e=u v \in M$, we say that $u$ and $v$ are twins, and write $u=t(v)$ (and so $v=t(u)$ ). For $S \subseteq V(M)$ we write $T(S)=\{t(v): v \in S\}$. These definitions depend on $M$, but this will be fixed throughout, so we suppress it in our notation.

Any edge that goes between $V(G) \backslash V(M)$ and $V(M)$ is called external.

We say that $M$ avoids a set $S$ of vertices if $V(M) \cap S=\emptyset$. We say that $M$ avoids a set $C$ of colors if $C(M) \cap C=\emptyset$. We say that $M$ fixes a set $E$ of edges if $E \subseteq M$.

Suppose $M$ and $M^{\prime}$ are rainbow matchings in $G$. We say $M$ and $M^{\prime}$ are $\lambda$-close if $\left|M^{\prime}\right|=|M|$ and $\left|M \triangle M^{\prime}\right| \leq \lambda$. Note that this is nonstandard terminology. (Standard usage would not include the condition $\left|M^{\prime}\right|=|M|$.)

For the remainder of the paper we fix $\varepsilon, k, n$, and $G$ as in the statement of Theorem 1.1. We also use an auxiliary variable $\alpha:=\varepsilon / 16$. We fix a rainbow matching $M$ in $G$ of maximum size and write $V_{0}=V(G) \backslash V(M)$. Let $C_{0}$ be the set of colors not used by $M$. We note that by maximality there are no $C_{0}$-edges contained in $V_{0}$. We suppose for a contradiction that $\left|C_{0}\right|>k$.

3. The reachability algorithm. We will describe an algorithm which builds a set of colors that we call reachable. Informally, these are the colors $c$ in $M$ such that we can robustly replace $c$ by some unused color $c_{0} \in C_{0}$ and obtain another rainbow matching $M^{\prime}$ with $\left|M^{\prime}\right|=|M|$. To achieve this we may need to make several other changes to the matching, but if the number of changes is bounded by some constant,

\footnotetext{
${ }^{2}$ We thank an anonymous referee for this comment, which requires some thought, as it is not immediately apparent that their result implies the bipartite version of ours.
} 
then we think of $c$ as reachable. The changes will consist of several flips along disjoint $M$-alternating paths (as in the classical matching algorithms).

As a first step, let us show that there are many edges in $M$ which we call "flexible," meaning they have several options to be directly replaced by a $C_{0}$-edge, with no further changes to the matching. This property is not as strong as the reachability property that we will define below, but in the analysis of reachability we will need to know that there are many flexible edges.

We let $E_{0}$ be the set of edges in $M$ which can be oriented from tail to head so that the number of external $C_{0}$-edges incident to the tail is at least $\alpha\left|C_{0}\right|$. (If both orientations work, then we choose one arbitrarily.) We let $S_{0}$ denote the set of heads of edges in $E_{0}$ (so the set of tails is the set $T\left(S_{0}\right)$ of twins of $S_{0}$ ). Then $F=C\left(E_{0}\right)$ is the set of flexible colors.

For any $v \in S_{0}$ there are at least $\alpha\left|C_{0}\right|$ choices of a $C_{0}$-edge $t(v) u$ with $u \in V_{0}$, and $M^{\prime}=M \backslash\{v t(v)\} \cup\{t(v) u\}$ is a rainbow matching with $\left|M^{\prime}\right|=|M|$. Note however that $u$ may be the same for all such $C_{0}$-edges.

LEMma 3.1. $|F| \geq \alpha n$.

Proof. For every $c \in C_{0}$, we note that there are at least $\varepsilon n$ external $c$-edges. Indeed, the total number of $c$-edges is at least $n+\varepsilon n$, there are none in $V_{0}$, and at most $n$ are contained in $V(M)$, as $|M|<n$ and the coloring is proper. Thus we have at least $\varepsilon n \cdot\left|C_{0}\right|$ external $C_{0}$-edges. Note that any vertex in $V(M)$ is incident to at most $\left|C_{0}\right|$ of these edges, and by definition of $F$, any vertex of $V(M) \backslash\left(S_{0} \cup T\left(S_{0}\right)\right)$ is incident to at most $\alpha\left|C_{0}\right|$ of them. Thus $\varepsilon n \cdot\left|C_{0}\right| \leq 2|F| \cdot\left|C_{0}\right|+2 n \cdot \alpha\left|C_{0}\right|$, so $|F| \geq\left(\frac{\varepsilon}{2}-\alpha\right) n \geq \alpha n$.

For more options of switches we need longer paths. For example, if an edge $e$ of $M$ is incident to many external $F$-edges, we may be able to use one of these edges instead of $e$, and then remove the corresponding $F$-edge from $M$ using its incident $C_{0}$-edges. We will be able to implement this idea for edges satisfying the following condition. For $c \in F$, we classify external $c$-edges as good or bad, where $e$ is good if $m_{c}$ (the $c$-edge in $M$ ) is incident to at least $\alpha\left|C_{0}\right| / 2$ external $C_{0}$-edges which share no endpoint with $e$.

Now we formulate our algorithm.

Algorithm 3.1. We iteratively define sets $E_{i}$ of "i-reachable" edges for $i \geq 1$. Each edge in $E_{i}$ will be assigned an orientation, from tail to head. We let $V_{i}$ denote the set of heads of edges in $E_{i}$ (so the set of tails is the set $T\left(V_{i}\right)$ of twins of $V_{i}$ ). We write $R_{i}=C\left(E_{i}\right)$ for the set of $i$-reachable colors.

We let $E_{1}$ be the set of edges in $M$ that can be oriented from tail to head such that the tail is incident to at least $\alpha|F| \operatorname{good} F$-edges.

For $i>1$ we let $E_{i}$ be the set of edges in $M \backslash \cup_{1 \leq j<i} E_{j}$ that can be oriented from tail $t$ to head such that for some $1 \leq j<i$ the number of $R_{j}$-edges tu with $u \in \cup_{j=0}^{i-1} V_{j}$ is at least $\alpha\left|R_{j}\right|$. (Note that for all $i \geq 1$ if both orientations work, then we choose one arbitrarily.)

We stop if $\left|E_{i}\right|<\alpha n$.

Let $q$ be such that the algorithm stops with $\left|E_{q+1}\right|<\alpha n$. As $|M|<n$, we have $q<1 / \alpha$.

Let $R$ be the set of reachable colors, that is, $R=\cup_{i=1}^{q} R_{i}$. We also write $V_{\text {reach }}=$ $\cup_{i=1}^{q} V_{i}$. 
Our next lemma shows that the first step of the algorithm finds some linear proportion of reachable colors.

LEMMA 3.2. $\left|R_{1}\right| \geq \alpha$.

Proof. First we show that there are few $F$-edges contained in $V_{0}$.

Claim 3.3. For any color $c \in F$, there can be at most one $c$-edge contained in $V_{0}$.

Proof. Indeed, fix $c \in F$, and suppose there exist two such edges, say, $e_{1}$ and $e_{2}$. Let $m_{c}=u t(u)$, where $u \in S_{0}$. Fix any external $C_{0}$-edge $e_{3}$ incident to $t(u)$ (by definition of $S_{0}$ ) and suppose without loss of generality that $e_{2} \cap e_{3}=\emptyset$. (We have $e_{1} \cap e_{2}=\emptyset$, as the coloring is proper.) Then $M^{\prime}=M \backslash\{u t(u)\} \cup\left\{e_{2}, e_{3}\right\}$ is a larger rainbow matching, which contradicts our choice of $M$.

We deduce that there are at least $\varepsilon|F| n / 2$ external $F$-edges. Indeed, the total number of $F$-edges is at least $(1+\varepsilon) n|F|$, of which at most $|F|$ are in $V_{0}$ (by Claim 3.3), and at most $|F| n$ are contained in $V(M)$ (as $|M|<n$ and the coloring is proper).

Claim 3.4. There are at least $\varepsilon|F| n / 4$ good external $F$-edges.

Proof. It suffices to show that for each $c \in F$ there are at most $2 / \alpha$ bad $c$-edges. (Then the number of bad $c$-edges is at most $2|F| / \alpha<\varepsilon|F| n / 4$.) To see this, fix $c \in F$, let $m_{c}=u t(u)$, where $u \in S_{0}$, and consider any external $c$-edge $v w$ that is bad with $w \in V_{0}$. There are at least $\alpha\left|C_{0}\right|$ external $C_{0}$-edges incident to $t(u)$ (by definition of $S_{0}$ ), of which at most $\alpha\left|C_{0}\right| / 2$ do not contain $w$ (by definition of "bad"), so $t(u) w$ has multiplicity at least $\alpha\left|C_{0}\right| / 2$ in $C_{0}$-edges. As the coloring is proper, there are at most $\left|C_{0}\right|$ (external) $C_{0}$-edges incident to $t(u)$, and each bad external $c$-edge determines a unique $w$ incident to at least $\alpha\left|C_{0}\right| / 2$ of them, so the claim follows.

On the other hand, by definition of $R_{1}$ there are at most $2\left|R_{1}\right||F|+2 n \cdot \alpha|F|$ external $F$-edges, so by Claim 3.4

$$
\varepsilon|F| n / 4 \leq 2\left|R_{1}\right||F|+2 n \cdot \alpha|F|,
$$

giving $\left|R_{1}\right| \geq \varepsilon n / 8-\alpha n=\alpha n$.

Our next definition and associated lemma justify our description of the colors in $R$ as "reachable." The lemma shows that they can be replaced in the matching, maintaining its size and being rainbow, while making only constantly many changes and avoiding certain proscribed sets of vertices, edges, and colors.

Definition 3.5. For $1 \leq i \leq q$ we say that $M$ is $\left(i, \lambda, \lambda^{\prime}, a\right)$-robust if for any rainbow matching $M^{\prime}$ which is $\lambda$-close to $M$, any $c \in R$ such that $m_{c}=v t(v) \in M^{\prime}$ with $v \in \cup_{j=1}^{i} V_{j}$, and any $E_{f i x} \subseteq M^{\prime} \backslash\left\{m_{c}\right\}$, any $V_{\text {avoid }} \subseteq V \backslash V\left(M^{\prime}\right)$, and $C_{\text {avoid }} \subseteq$ $C \backslash C\left(M^{\prime}\right)$, each of size at most a, there is a rainbow matching $M^{\prime \prime}$ in $G$ such that

(1) $c \notin C\left(M^{\prime \prime}\right)$ and $v \notin V\left(M^{\prime \prime}\right)$,

(2) $M^{\prime \prime}$ is $\lambda^{\prime}$-close to $M$,

(3) $M^{\prime \prime}$ fixes $E_{\text {fix }}$ and avoids $C_{\text {avoid }}$ and $V_{\text {avoid }}$.

We will apply the following statement only in the case $i=q$, but we formulate it for all $i$ to facilitate an inductive proof. We write $f(i)=3 \cdot 2^{i-1}-2$.

LEMmA 3.6. Let $1 \leq i \leq q$ and $0 \leq \lambda \leq 2^{15 / \varepsilon-i / 15}$. Then $M$ is $(i, \lambda, \lambda+f(i), 2(q-$ $i+1)$ )-robust. 
Proof. Let $M^{\prime}, c, v, E_{f i x}, V_{\text {avoid }}$, and $C_{\text {avoid }}$ be given as in Definition 3.5. We will show by induction on $i$ that there is a rainbow matching $M^{\prime \prime}$ satisfying (1), (2), and (3) of Definition 3.5.

First we consider the base case $i=1$. We consider the set $\mathcal{T}$ of all pairs $(v t(v) w, u t(u) z)$ of 2-edge paths, where $t(v) w$ is a good $F$-edge, $w$ and $z$ are distinct and in $V_{0}$, and we have $c(t(v) w)=c(u t(u))$ and $c(t(u) z) \in C_{0}$. If $v t(v), u t(u)$ also appear in $M^{\prime}$ (a priori they are from $M$ ), we will obtain $M^{\prime \prime}$ from $M^{\prime}$ by removing $v t(v)$ and $u t(u)$ and adding $t(v) w$ and $t(u) z$. Note that any such $M^{\prime \prime}$ is $(\lambda+4)$-close to $M$, as required for (2). There are various constraints that need to be satisfied for $M^{\prime \prime}$ to be a rainbow matching satisfying (1) and (3); we will see below that $\mathcal{T}$ is large enough so that we can pick an element of $\mathcal{T}$ such that the matching derived from it satisfies these constraints.

First we show that $|\mathcal{T}| \geq \alpha^{3}\left|C_{0}\right| n / 2$. To see this, note that as $v \in V_{1}$ there are at least $\alpha|F|$ good external $F$-edges incident to $t(v)$. For each such edge $t(v) w$, there is an edge in $M$ of the same color, which we can label as $u t(u)$ so that there are at least $\alpha\left|C_{0}\right| / 2$ external $C_{0}$-edges $t(u) z$ with $z \neq w$. Hence, by Lemma 3.1 we get that $|\mathcal{T}| \geq \alpha|F| \cdot \alpha\left|C_{0}\right| / 2 \geq \alpha^{3}\left|C_{0}\right| n / 2$.

Next we estimate how many configurations in $\mathcal{T}$ are forbidden by the various constraints described above.

We start by considering the constraints on $w$, namely, $w \in V_{0}^{\prime}$ and $w \notin V_{\text {avoid }}$. These forbid at most $2 \lambda+2 q$ choices of $w$. For each such $w$, there are at most $n / k$ choices of an $F$-edge $t(v) w$, which determines $u t(u) \in M$ with $c(u t(u))=c(t(v) w)$, and then there are at most $\left|C_{0}\right|$ choices for a $C_{0}$-edge $t(u) z$. Thus we forbid at most $(2 \lambda+2 q)\left|C_{0}\right| n / k$ configurations.

We also have the same constraints on $z$, i.e., $z \in V_{0}^{\prime}$ and $z \notin V_{\text {avoid. Again, }}$ this forbids at most $2 \lambda+2 q$ choices of $z$. For each such $z$, there are at most $\left|C_{0}\right|$ choices for a $C_{0}$-edge $t(u) z$, which determines $u t(u) \in M$, and then at most one edge $t(v) w$ with the same color. Thus we forbid at most $(2 \lambda+2 q)\left|C_{0}\right|$ configurations.

Now we consider the constraints on $u t(u)$, namely, $u t(u) \in M^{\prime}$ and $u t(u) \notin E_{f i x}$. These forbid at most $\lambda+2 q$ choices of $u t(u)$. For each such $u t(u)$, there is at most one edge $t(v) w$ with the same color, and at most $\left|C_{0}\right|$ choices for $t(u) z$, so we forbid at most $(\lambda+2 q)\left|C_{0}\right|$ configurations.

For $t(v) w$ we have the constraint $c(t(v) w) \in C\left(M^{\prime}\right)$, which forbids at most $\lambda$ edges $t(v) w$. This fixes $u t(u) \in M$ with the same color, and then there are at most $\left|C_{0}\right|$ choices for $t(u) z$, so we forbid at most $\lambda\left|C_{0}\right|$ configurations.

Finally, for $t(u) z$ we have the constraint $c(t(u) z) \notin C_{\text {avoid }} \cup\left(C\left(M^{\prime}\right) \backslash C(M)\right)$. There are at most $2 q+\lambda$ choices for $c^{\prime} \in C_{\text {avoid }} \cup\left(C\left(M^{\prime}\right) \backslash C(M)\right)$, then at most $n$ choices for a $c^{\prime}$-edge $t(u) z$, which determines $u t(u)$ and then at most one edge $t(v) w$ with the same color, so we forbid at most $(2 q+\lambda) n$ configurations.

The total number of forbidden configurations is at most $2(\lambda+m)\left|C_{0}\right|(2+n / k)+$ $(2 q+\lambda) n<\alpha^{3}\left|C_{0}\right| n / 2 \leq|\mathcal{T}|$, as $\lambda / k \leq 2^{-5 / \varepsilon}<10^{-6} \varepsilon^{3}$, so $2(\lambda+m)\left|C_{0}\right|(2+n / k)<$ $\alpha^{3}\left|C_{0}\right| n / 4$ and $\alpha^{3}\left|C_{0}\right| n / 4>\alpha^{3} k n / 4>(2 q+\lambda) n$. We can therefore pick a configuration in $\mathcal{T}$ that satisfies all the above constraints, which completes the proof of the base case.

Now suppose $i>1$. As $v \in V_{i}$, there is $1 \leq j<i$ such that the number of $R_{j^{-}}$ edges $t(v) w$ with $w \in \cup_{j=0}^{i-1} V_{j}$ is at least $\alpha\left|R_{j}\right| \geq \alpha^{2} n$. (Note that $\left|R_{j}\right|=\left|E_{j}\right| \geq \alpha n$; otherwise the algorithm would have stopped.) We will consider two cases according to whether most of these edges go to $V_{0}$. In each case we note by the induction hypothesis that $M$ is $(j, \lambda, \lambda+f(j), 2(q-j+1))$-robust. 
Suppose first that at least $\alpha^{2} n / 2$ of these $R_{j}$-edges $t(v) w$ have $w \in V_{0}$. Then we can fix such an edge $t(v) w$ with $c^{\prime}=c(t(v) w) \in R_{j}$ such that $w \in V_{0}^{\prime} \backslash V_{\text {avoid }}$ and $m_{c^{\prime}} \in M^{\prime} \backslash E_{f i x}$. (The first condition forbids at most $(2 q+2 \lambda) n / k$ choices and the second at most $2 q+\lambda$ choices.)

By induction hypothesis, we can apply Definition 3.5 applied to $m_{c^{\prime}}$ in $M^{\prime}$ with sets $E_{\text {fix }}^{\prime}:=E_{f i x} \cup\{v t(v)\}, V_{\text {avoid }}^{\prime}:=V_{\text {avoid }} \cup\{w\}$, and $C_{\text {avoid }}^{\prime}:=C_{\text {avoid }}$, each of size at most $2(q-i+1)+1<2(q-j+1)$, and we obtain a rainbow matching $M_{1}$ which is $(\lambda+f(j))$-close to $M$, satisfies (3), contains $v(t) v$, and avoids the color $c^{\prime}$ and vertex w. As $\lambda+f(j)+2 \leq \lambda+f(i)$, we see that $M^{\prime \prime}=M_{1} \backslash\{v t(v)\} \cup\{t(v) w\}$ is as required to complete the proof in this case.

It remains to consider the case that at least $\alpha^{2} n / 2$ of the $R_{j}$-edges $t(v) u$ have $u \in \cup_{j=1}^{i-1} V_{j}$. We can fix such an edge $t(v) u$ with $c(t(v) u)=c_{1} \in R_{j}$ such that $c(u t(u))=c_{2} \in R_{j^{\prime}}$ with $1 \leq j^{\prime} \leq i-1$ and $u t(u) \in M^{\prime} \backslash E_{f i x}$ (this forbids at most $(2 q+\lambda) n / k$ choices), and $m_{c_{1}} \in M^{\prime} \backslash E_{f i x}$ (which forbids at most $2 q+\lambda$ choices).

By induction hypothesis, we can apply Definition 3.5 to $m_{c_{1}}$ in $M^{\prime}$ with sets $E_{f i x}^{\prime}:=E_{f i x} \cup\{v t(v), u t(u)\}, V_{\text {avoid }}^{\prime}:=V_{\text {avoid}}$, and $C_{\text {avoid }}^{\prime}:=C_{\text {avoid }}$, each of size at most $2(q-i+1)+2 \leq 2(q-j+1)$, and we obtain a rainbow matching $M_{1}$ which is $(\lambda+f(j))$-close to $M$, satisfies (3), contains $v(t) v$ and $u t(u)$, and avoids the color $c_{1}$.

By induction hypothesis, we can apply Definition 3.5 to $m_{c_{2}}=u t(u) \in M_{1}$ with sets $E_{f i x}^{\prime \prime}:=E_{f i x} \cup\{v t(v)\}, V_{\text {avoid }}^{\prime \prime}:=V_{\text {avoid }}$, and $C_{\text {avoid }}^{\prime \prime}:=C_{\text {avoid }} \cup\left\{c_{1}\right\}$, noting that $\lambda+f(j) \leq 2^{15 / \varepsilon-i / 15}+2^{1+1 / \alpha} \leq 2^{15 / \varepsilon-j^{\prime} / 15}$, as $\alpha=\varepsilon / 12, j^{\prime} \leq i-1, i \leq m<1 / \alpha$, and $\varepsilon<10^{-3}$. Thus we obtain a rainbow matching $M_{2}$ which is $\left(\lambda+f(j)+f\left(j^{\prime}\right)\right)$ close to $M$, satisfies (3), contains $v(t) v$, and avoids the color $c_{1}$ and the vertex $u$. As $\lambda+f(j)+f\left(j^{\prime}\right)+2 \leq \lambda+f(i)$, we see that $M^{\prime \prime}=M_{2} \backslash\{v t(v)\} \cup\{t(v) u\}$ is as required to complete the proof.

4. Structure and counting. Continuing with the proof strategy outlined above, we now use the maximality assumption on $M$ to prove the following structural properties of $G$, which we will then combine with a counting argument to obtain a contradiction to the assumption that $\left|C_{0}\right|>k$, thus completing the proof of Theorem 1.1.

In the proof of the following lemma we repeatedly use Lemma 3.6 in the case $i=q$, i.e., that $M$ is $(q, \lambda, \lambda+f(q), 2)$-robust for any $0 \leq \lambda \leq 2^{15 / \varepsilon-q / 15}$. Here we recall that $R=\cup_{i=1}^{q} R_{i}$ and $q<1 / \alpha=12 / \varepsilon$, so Definition 3.5 is applicable whenever $0 \leq \lambda \leq 2^{14 / \varepsilon}$.

LEMMA 4.1.

(C1) Any $v \in V_{\text {reach }}$ is not incident to an external $R$-edge.

(C2) Any two $u, v \in V_{\text {reach }}$ are not adjacent by an $R$-edge.

(C3) There are no $R$-edges contained in $V_{0}$.

Proof. (C1): Suppose $v$ is incident to an external $R$-edge, say, $w \in V_{0}$ and $c=$ $c(v w) \in R$. We can apply Lemma 3.6 to $v t(v)$ in $M^{\prime}=M$ with sets $E_{f i x}=\left\{m_{c}\right\}$ and $V_{\text {avoid }}=\{w\}, C_{\text {avoid }}=\emptyset$ and obtain a rainbow matching $M_{1}$ that is $f(q)$-close to $M$, contains $m_{c}$ and avoids $v$ and $w$. As $f(q)<2^{14 / \varepsilon}$, we can apply Lemma 3.6 again to $m_{c}$ in $M_{1}$ with sets $E_{f i x}=C_{\text {avoid }}=\emptyset$ and $V_{\text {avoid }}=\{v, w\}$, obtaining a rainbow matching $M_{2}$ that is $2 f(q)$-close to $M$ and avoids the vertices $v, w$ and the color $c$. Now $M_{2} \cup\{u w\}$ is a larger rainbow matching than $M$, which is a contradiction.

(C2): Suppose for a contradiction that we have $u, v \in V_{\text {reach }}$ and an edge $u v$ with $c(u v)=c_{3} \in R$. Let $c_{1}=c(v t(v))$ and $c_{2}=c(u t(u))$. Then $c_{1}$ and $c_{2}$ are also in $R$. 
Applying Lemma 3.6 to $v t(v)$ in $M^{\prime}=M$ with sets $E_{f i x}=\left\{u t(u), m_{c_{3}}\right\}$ and $V_{\text {avoid }}=C_{\text {avoid }}=\emptyset$, we obtain a rainbow matching $M_{1}$ that is $f(q)$-close to $M$, contains $u t(u)$ and $m_{c_{3}}$, and avoids $v$.

Applying Lemma 3.6 again to $u t(u)$ in $M_{1}$ with sets $E_{f i x}=\left\{m_{c_{3}}\right\}, V_{\text {avoid }}=\{v\}$, and $C_{\text {avoid }}=\emptyset$, we obtain a rainbow matching $M_{2}$ that is $2 f(q)$-close to $M$, contains $m_{c_{3}}$, and avoids $u$ and $v$.

Applying Lemma 3.6 a final time to $m_{c_{3}}$ in $M_{2}$ with $V_{\text {avoid }}=\{u, v\}$ and $E_{f i x}=$ $C_{\text {avoid }}=\emptyset$, noting that $2 f(q)<2^{14 / \varepsilon}$, we obtain a rainbow matching $M_{3}$ that is $3 f(q)$-close to $M$ and avoids the vertices $u$ and $v$ and the color $c_{3}$.

Now $M_{3} \cup\{u v\}$ is a larger rainbow matching than $M$, which is a contradiction.

(C3): We omit the proof as it is very similar to that of the first two statements.

Proof of Theorem 1.1. Recalling that $M$ is a maximum rainbow matching in $G$, we write $V_{0}=V(G) \backslash V(M)$ and suppose for a contradiction that $|M|<n-k$. Recalling also that the reachable colors are $R=\cup_{i=1}^{q} R_{i}$, we write $V_{\text {reach }}=\cup_{i=1}^{q} V_{i}$, and the algorithm stopped with $\left|V_{q+1}\right|<\alpha n$.

As every color appears at least $(1+\varepsilon) n$ times the number of $R$-edges is at least $|R|(1+\varepsilon) n$. By Lemma 4.1 they are all incident to $V(M) \backslash V_{\text {reach }}$. Write

$$
V^{*}=T\left(V_{\text {reach }}\right) \cup V_{q+1} \cup T\left(V_{q+1}\right) \text { and } V^{\prime}=V(M) \backslash\left(V_{\text {reach }} \cup V^{*}\right) .
$$

At most $\left|V^{*}\right||R|<(|R|+2 \alpha n)|R|$ of the $R$-edges are incident to $V^{*}$, so at least $|R|(1+\varepsilon) n-(|R|+2 \alpha n)|R|>|R|\left(\left|V^{\prime}\right|+\varepsilon n\right) / 2$ are incident to $V^{\prime}$ but not to $V^{*}$.

By definition of our algorithm, for each $v$ in $V^{\prime}$ the number of $R$-edges $v u$ with $u \in V_{0} \cup V_{\text {reach }}$ is at most $\alpha|R|$. All remaining $R$-edges are contained in $V^{\prime}$, and they number at least $|R|\left(\left|V^{\prime}\right|+\varepsilon n\right) / 2-\alpha|R|\left|V^{\prime}\right|>|R|\left|V^{\prime}\right| / 2$. But the coloring is proper, so there are at most $\left|V^{\prime}\right| / 2$ edges of any color contained in $V^{\prime}$. This contradiction completes the proof.

5. Conclusion. A similar result to Theorem 1.1 was very recently obtained independently by Gao et al. [4]. Their result is closer than ours to the spirit of Conjecture 1.2 , as they obtain a full rainbow matching (whereas we allow a constant number of unused colors). However, our results are incomparable, as they need a stronger bound on edge multiplicities, namely, $\sqrt{n} / \log ^{2} n$. Our algorithm is deterministic, whereas theirs is randomized, and the analysis of our algorithm is simpler. In any case, the two approaches are very different, so we think that it will be valuable to pursue both sets of ideas, and perhaps also the approach of Pokrovskiy, in making further progress on Conjecture 1.2.

Acknowledgment. We thank the anonymous referees for helpful comments.

\section{REFERENCES}

[1] R. Aharoni and E. Berger, Rainbow matchings in r-partite r-graphs, Electron. J. Combin., 16 (2009).

[2] J. BARÁt, A. GyÁrfás, AND G.N. SÁRKÖZy, Rainbow Matchings in Bipartite Multigraphs, arXiv:1505.01779.

[3] R. A. Brualdi and H. J. Ryser, Combinatorial Matrix Theory, Cambridge University Press, Cambridge, 1991.

[4] P. Gao, R. Ramadurai, I. Wanless, and N. Wormald, Full Rainbow Matchings in Graphs and Hypergraphs, arXiv:1709.02665.

[5] P. Hatami and P. W. Shor, A lower bound for the length of a partial transversal in a Latin square, J. Combin. Theory Ser. A, 115 (2008), pp. 1103-1113.

[6] A. Pokrovskiy, An Approximate Version of a Conjecture of Aharoni and Berger, arXiv:1609.06346. 
[7] A. Pokrovskiy and B. Sudakov, A Counterexample to Stein's Equi-n-square Conjecture, arXiv:1711.00429.

[8] H. Ryser, Neuere probleme in der kombinatorik, Vortrage über Kombinatorik, Oberwolfach, 1967, pp. 69-91.

[9] S. K. Stein, Transversals of Latin squares and their generalizations, Pacific J. Math., 59 (1975), pp. 567-575.

[10] I. M. WANLESS, Transversals in Latin squares: A Survey, Surveys in Combinatorics, Cambridge University Press, Cambridge, 2011.

Copyright (c) by SIAM. Unauthorized reproduction of this article is prohibited. 